\title{
Cambios en la cubierta terrestre a través de los mapas ESA-CCI-LC (2000-2015), Ixtacamaxtitlán, Puebla
}

\author{
Land cover changes through ESA-CCI-LC maps (2000-2015) in \\ Ixtacamaxtitlán, Puebla
}

\author{
Itzel Arroyo-Ortegal; Víctor Tamaríz-Flores 1,6; Eduardo Torres-Ramírez ${ }^{1,3}$, Anabella Handal-Silva1,4, \\ Edith Chávez-Bravo1,5; Virginia Cervantes-Gutiérrez² y Rosalía Castelán-Vega*1,6
}

\begin{abstract}
1 Benemérita Universidad Autónoma de Puebla. Posgrado en Ciencias Ambientales. Instituto de Ciencias. Puebla, Puebla, México.

2 Universidad Autónoma Metropolitana-Xochimilco. Departamento: El hombre y su ambiente. Ciudad de México, México.

3 Benemérita Universidad Autónoma de Puebla. Centro de Química. Puebla, Puebla, México.
\end{abstract}

4 Benemérita Universidad Autónoma de Puebla. Departamento de Biología y Toxicología de la

Reproducción. Puebla, Puebla, México.

5 Benemérita Universidad Autónoma de Puebla.

Centro de Investigaciones en Ciencias

Microbiológicas. Puebla, Puebla, México.

6 Benemérita Universidad Autónoma de Puebla.

Departamento de Investigación en Ciencias

Agrícolas. Puebla, Puebla, México.

\section{RESUMEN}

La determinación de cambios en la cubierta terrestre (CT) es importante para entender la dinámica de los ecosistemas. En este estudio se analizaron los cambios espaciales y temporales en el municipio de Ixtacamaxtitlán, Puebla durante el periodo 2000-2015, utilizando los mapas globales de la Agencia Espacial Europea ESA-CCI-LC. Para ello, se determinó la matriz de cambios, la tasa anual de cambio y la probabilidad de permanencia, así como la precisión del mapa de CT 2015. Los resultados indican que las pérdidas se concentraron en las coberturas arboladas y pastizales (0.27\% y 8.83\%, respectivamente), mientras que las ganancias se dieron en tierras de cultivo y zonas urbanas (6.9\% y $8.11 \%$, respectivamente). La mayoría de los cambios se observaron en las zonas de fácil acceso ubicadas en la porción centro-norte del municipio. De las 1962 ha que presentaron cambios entre categorías, en 87.4\% el cambio fue negativo y en $12.6 \%$ fue positivo, destacando la transición de pastizales y superficies arboladas hacia tierras de cultivo, causadas por diversos procesos socioeconómicos presentes en la zona como marginación, migración, acceso a programas gubernamentales, expansión de áreas agrícolas y diversificación de cultivo. El mapa de CT 2015 mostró una alta precisión a escala subnacional (85.64\%) lo que revela que este conjunto de datos es confiable para el estudio de las transformaciones en el territorio.

PALABRAS ClAVE: conversión de tierras, degradación de tierras, matriz de cambios, probabilidad de permanencia, SIG, tasa anual de cambio.

\section{ABSTRACT}

The determination of Land Cover (LC) changes is important to understand ecosystem dynamics. In this study, spatial-temporal changes were analyzed in the municipality of Ixtacamaxtitlán, Puebla during the period 2000-2015 using European Space Agency (ESA) Climate Change Initiative Land Cover (CCI-LC) global maps. For this purpose, the change matrix, the annual change rate, and the permanence probability were determined, as well as the accuracy of the LC 2015 map. The results show that losses were observed in forests and grasslands ( $0.27 \%$ and $8.83 \%$, respectively), while gains occurred in croplands and urban areas (6.9\% and $8.11 \%$, respectively). Most of the changes were observed in the easy-access areas located in the north-central portion of the municipality. Of the 1962 ha that showed changes between categories, $87.4 \%$ was negative and $12.6 \%$ was positive, highlighting the transition from grasslands and forest lands to cropland, caused by various socio-economic processes in the area such as marginalization, migration, access to government programs, expansion of agricultural areas and crop diversification. The map of LC 2015 showed a high precision at the subnational level (85.64\%) which reveals that this data set is reliable for the study of the transformations in the territory.

KEYWORDS: land conversion, land degradation, change matrix, permanence probability, GIS, annual change rate. 


\section{INTRODUCCIÓN}

La determinación de los cambios en la cubierta terrestre (CT) tiene un papel relevante para el entendimiento de las dinámicas y procesos en los ecosistemas que se dan a través del tiempo y el espacio, al ofrecer un primer indicio de la reducción o aumento de la vegetación, la fragmentación del hábitat y el uso y conversión de tierras (United Nations Convention to Combat Desertification [UNCCD] 2016; Orr et al., 2017; Mousivand y Arsanjani, 2019). Los cambios producidos son reconocidos como un factor implicado en problemáticas ambientales como la degradación de tierras, la pérdida de la biodiversidad y el aumento de gases de efecto invernadero (Feddema et al., 2005; Grekousis, Mountrakis y Kavouras, 2015; Jiang y Yu, 2019).

La combinación derivada del uso de teledetección y sistemas de información geográfica (SIG), es un recurso efectivo que permite la identificación de dichos cambios, así como su análisis y monitoreo a bajo costo (Guidigan, Sanou, Ragatoa, Fafa y Mishra, 2019). Sin embargo, pese a que los datos satelitales disponibles son abundantes y se han desarrollado diversos productos y herramientas para estimar los cambios producidos sobre la superficie de la Tierra, ha sido desafiante mantener un mapeo continuo y series temporales anuales durante largos periodos, que conserven la misma metodología de principio a fin, por lo que la determinación de los cambios en la CT presenta inconsistencias (Grekousis et al., 2015; Liu et al., 2018; Jiang y Yu, 2019).

En este sentido, existen diferencias entre precisión, diseño del sensor, resolución, procedimiento y esquema de clasificación utilizado en los productos disponibles, lo que dificulta la comparación de un conjunto de datos (clases de $\mathrm{CT}$, área y su distribución espacial) de una misma zona en un tiempo determinado, o con otras regiones (Fritz et al., 2011; Jiang y Yu, 2019). Esta problemática se acentúa a escalas menores, debido a la diversidad de clasificaciones de uso de suelo y cubierta vegetal que existen a escalas regionales y nacionales.

Recientemente la Agencia Espacial Europea (ESA, por sus siglas en inglés) lanzó la Iniciativa de Cambio Climático
(CCI, por sus siglas en inglés), con el objetivo de proporcionar un conjunto de datos de CT (LC, por sus siglas en inglés) que fueran estables y completos, pero, sobre todo, que tuvieran una consistencia temporal (Li et al., 2016). El producto generado con dicha iniciativa (llamado ESA-CCI-LC) ofrece una resolución espacial más alta en comparación con la que ofrecen la mayoría de los productos a escala global, así como una resolución temporal mayor, con intervalos de un año y un abanico completo de tipos de cobertura, lo que permite detectar los cambios que se producen en el tiempo a diversas escalas (Li et al., 2018; Mousivand y Arsanjani, 2019).

En México, está disponible el conjunto de datos de uso de suelo y vegetación realizado por el Instituto Nacional de Estadística y Geografía (Inegi), sin embargo, la temporalidad de las series (I-VI) varía (1997, 2001, 2005, 2009, 2013 y 2016); así mismo las clases utilizadas en cada serie son disímiles (Peralta-Carreta, Gallardo-Cruz, Solórzano y Hernández-Gómez, 2019), por lo que no es posible realizar una cuantificación detallada sobre el cambio en las categorías de CT o conocer una tendencia anual de cambios en una zona determinada.

Por lo anterior, existe la necesidad de utilizar conjuntos de datos que presenten coherencia temporal y una clara compatibilidad en las categorías de CT que permitan la comparación entre regiones, y que a su vez faciliten la interpretación de los resultados, esto con la finalidad de comprender las interacciones entre las actividades antropogénicas y el ambiente que puedan generar estrategias para mitigar los impactos negativos que se produzcan (Dewan, Yamaguchi y Rahman, 2012; Guidigan et al., 2019).

La región de la Sierra Norte de Puebla ha presentado un constante deterioro ambiental; particularmente en el municipio de Ixtacamaxtitlán (Sánchez, 2015). A la fecha, estos cambios no han sido cuantificados para conocer la magnitud del deterioro ambiental presentado durante los últimos años. 


\section{OBJETIVOS}

El presente estudio planteó como objetivos identificar las categorías de cubierta terrestre presentes en el municipio de Ixtacamaxtitlán, Puebla, México y su distribución, así como analizar los cambios espaciales y temporales producidos en el periodo 2000 a 2015 mediante los mapas globales de ESA-CCI-LC.

\section{MATERIALES Y MÉTODOS}

\section{Área de estudio}

El municipio de Ixtacamaxtitlán se localiza al norte del estado de Puebla, México (Fig. 1), entre los paralelos $19^{\circ}$ $27^{\prime}$ y $19^{\circ} 45^{\prime}$ de latitud norte y los meridianos $97^{\circ} 41^{\prime}$ y $98^{\circ}$ 03' de longitud oeste. Tiene una superficie aproximada de 56767 ha, su altitud varía entre 2000 m y 3400 m s.n.m. El clima es templado y semifrío subhúmedo con lluvias en verano. La precipitación media anual del municipio es de
$800 \mathrm{~mm}$ y la temperatura varía entre $12{ }^{\circ} \mathrm{C}$ y $18{ }^{\circ} \mathrm{C}$ (Inegi, 2009). Los suelos con mayor cobertura en la zona son litosoles, regosoles y feozems; en menor medida se encuentran zonas con suelos del tipo andosol, cambisol y luvisol (Inegi, 2014).

El municipio concentra sus principales actividades económicas en agrícolas, pecuarias y forestales (Secretaría de Medio Ambiente y Recursos Naturales [Semarnat], 2013). De acuerdo con la Secretaría de Medio Ambiente y Recursos Naturales (Semarnat, 2013), a escala estatal, Ixtacamaxtitlán ocupa el cuarto lugar con mayor superficie de vegetación primaria de bosque de coníferas (12.62\%); así mismo, alrededor de $40 \%$ del municipio está compuesto por zonas forestales con vegetación primaria y secundaria de bosque de pino, oyamel, táscate y pino-encino, así como áreas de matorrales desértico rosetófilo que se desarrollan sobre elevaciones de rocas sedimentarias.

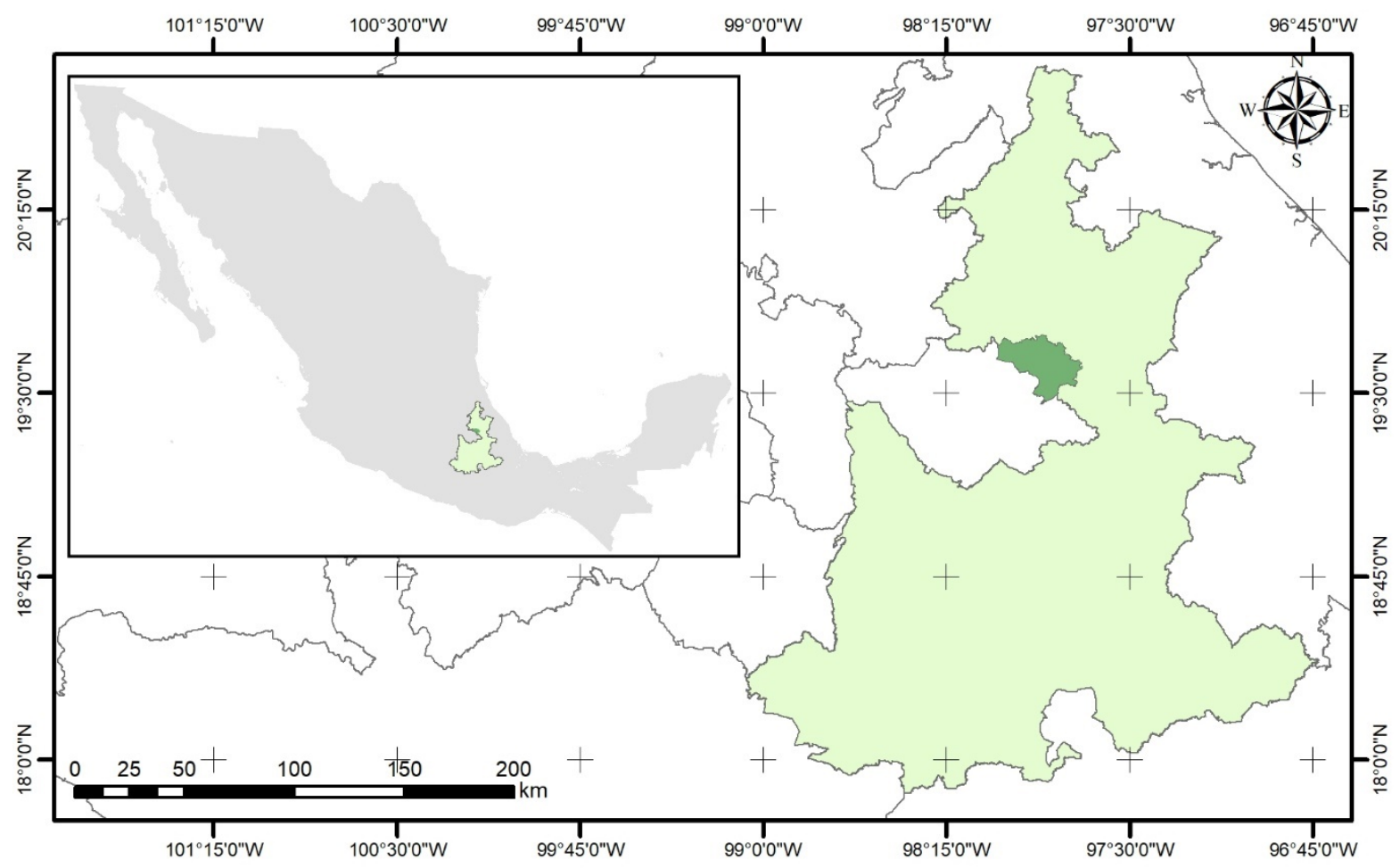

FIGURA 1. Localización del municipio de Ixtacamaxtitlán, Puebla, México. Fuente: Elaboración propia con información de Inegi (2009). 


\section{Materiales}

En esta investigación se utilizó el conjunto de datos de la Iniciativa contra el Cambio Climático de la Agencia Espacial Europea (ESA-CCI-LC) (UNCCD, 2018); el cual consiste en mapas de series temporales anuales de la CT correspondientes al periodo de 1992 a 2015, a una resolución de 300 m (Defourny et al., 2017; Li et al., 2018; Liu et al., 2018; Gichenje y Godinho, 2018; UNCCD, 2018).

De este conjunto de datos, se delimitó el polígono municipal de la zona de estudio de cada uno de los años del periodo de investigación (2000 a 2015). La información cartográfica se exportó a formato raster para su análisis y se procesó a través del programa ArcMap 10.2.2 (Environmental Systems Research Institute [ESRI], 2014).

\section{Categorías de análisis}

La clasificación original de CT que se empleó dentro del conjunto de datos de ESA-CCI-LC, fue la de Sistemas de
Clasificación de la Cubierta de la Tierra (LCCS, por sus siglas en inglés) estándar desarrollado por la Organización Mundial de las Naciones Unidas para la Alimentación y la Agricultura (FAO, 2016). Esta clasificación cuenta con 38 categorías que son aplicables para todo el planeta. En este estudio fueron alineadas con las siete categorías de uso de las tierras proporcionadas por el Panel Intergubernamental de Cambio Climático (IPCC, 2006) y la Convención de las Naciones Unidas de Lucha contra la Desertificación (UNCCD, 2018) con base en los criterios de la tabla 1, las cuales son las recomendadas para evaluar la degradación de las tierras a escala mundial (Programa Mundial de Apoyo [PMA], 2016; Orr et al., 2017).

Por último, se cuantificó el área total de cada una de las categorías de CT en los 16 mapas anuales obtenidos con la finalidad de determinar los cambios que se presentaron en el periodo de estudio.

TABLA 1. Correspondencia entre las categorías de CCI-LC, ESA y las categorías de CT UNCCD presentes en la zona de estudio.

\begin{tabular}{|c|c|c|c|}
\hline $\begin{array}{l}\text { Denominación } \\
\text { UNCCD }\end{array}$ & $\begin{array}{l}\text { Código } \\
\text { UNCCD }\end{array}$ & $\begin{array}{l}\text { Código } \\
\text { ESA-CCI-LC }\end{array}$ & Denominación ESA-CCI-LC \\
\hline \multirow{3}{*}{$\begin{array}{l}\text { Superficies } \\
\text { arboladas }\end{array}$} & \multirow{3}{*}{1} & 60 & Cobertura arbórea, hoja ancha, caduca, cerrada a abierta (>15\%). \\
\hline & & 70 & Cobertura arbórea, hoja acicular, perenne, cerrada a abierta (>15\%). \\
\hline & & 90 & Cobertura arbórea, hojas mixtas (ancha y acicular). \\
\hline \multirow{2}{*}{ Pastizales } & \multirow{2}{*}{2} & 120 & Monte bajo/matorral \\
\hline & & 130 & Pastizales. \\
\hline \multirow[b]{2}{*}{ Zonas de cultivo } & \multirow[b]{2}{*}{3} & 11 & Cobertura herbácea. \\
\hline & & 40 & $\begin{array}{l}\text { Mosaico en vegetación natural (cobertura arbórea, arbustiva y } \\
\text { herbácea) (>50\%) / tierras de cultivo }(<50 \%) \text {. }\end{array}$ \\
\hline $\begin{array}{l}\text { Superficies } \\
\text { artificiales }\end{array}$ & 5 & 190 & Asentamientos humanos. \\
\hline
\end{tabular}

Fuente: Elaboración propia con información de Programa Mundial de Apoyo (PMA), 2016 y United Nations Convention to Combat Desertification (UNCCD), 2018 


\section{Tasas de cambio anual}

Para conocer el porcentaje de cambio de la superficie al inicio de cada año se determinaron las tasas de cambio por categoría tomando en cuenta el periodo de inicio (2000) y final (2015) por medio de la siguiente ecuación (FAO, 1996):

$$
t=1-\left(\frac{S_{2}}{S_{1}}\right)^{\frac{1}{n}}
$$

donde:

$t=$ tasa de cambio

$S_{1}=$ superficie de uso del suelo en el tiempo inicial

$S_{2}=$ superficie de uso del suelo en el tiempo final

$n=$ amplitud del periodo evaluado

\section{Matriz de cambios}

Los cambios en la superficie terrestre se determinaron a partir de la construcción de una matriz de cambios (Pontius, Shusas y McEachern, 2004), la cual se generó por medio de la intersección de los datos raster obtenidos de los años 2000 y 2015, esta información se procesó en el módulo Raster Calculator del programa ArcGis 10.2.2 y, mediante algebra de mapas, se realizó la suma de los valores correspondientes a las capas. Esta matriz muestra los valores finales obtenidos que representan transiciones en la CT (CamachoSanabria, Camacho-Sanabria, Balderas-Plata y SánchezLópez, 2017).

\section{Cambios y flujos}

Con los datos obtenidos se generó un mapa de zonas que representaron cambios de CT del año 2000 a 2015, así como un mapa de flujos de CT del mismo periodo. Los cambios se consideraron positivos o negativos cuando pasaron de una a categoría a otra bajo los criterios mostrados en la figura 2. Los flujos por su parte fueron considerados como ganancias o pérdidas de cada categoría de CT presentes en la superficie (UNCCD, 2018).

\section{Probabilidad de permanencia}

A partir de la matriz de cambios se obtuvo la probabilidad de permanencia y transición de cada categoría. La cual, se determinó por medio de la división de cada una de las celdas de la matriz de cambios por categoría, entre la superficie total de la categoría analizada y es proporcional a la superficie remanente de la misma categoría en el periodo estudiado. La expresión matemática utilizada fue (Sánchez, Bocco, Fuentes y Velázquez, 2004):

$$
P i j=\frac{S i j(t 0)}{S j(t 1)}
$$

donde:

$P i j=$ probabilidad de permanencia y cuya sumatoria por categoría en el año 2000 es igual a 1

Sij = superficie de " $i j$ " de la matriz de cambios de CT en el año 2000

$S j=$ superficie de la categoría “ $j$ ” en el año 2015

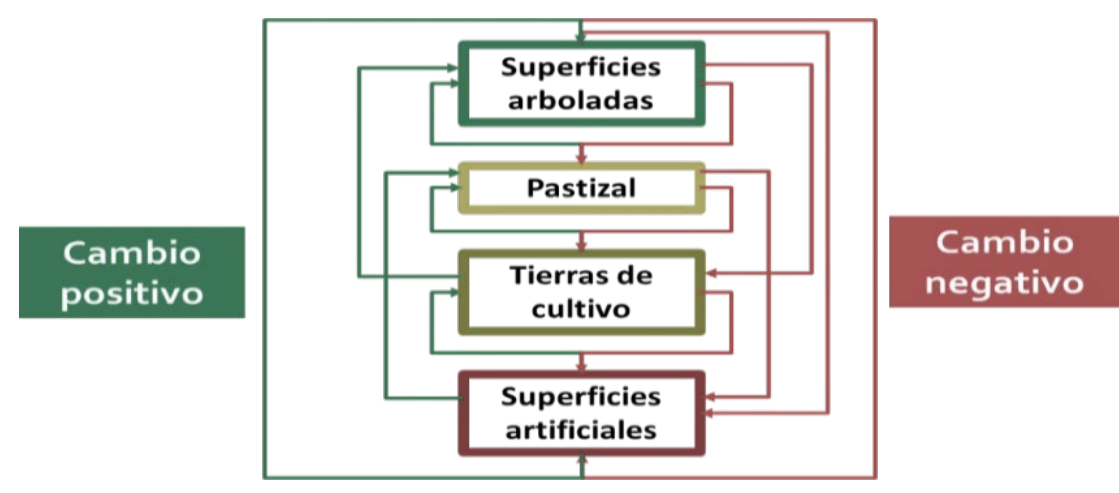

FIGURA 2: Diagrama de cambios de cubierta terrestre presentes en el municipio de Ixtacamaxtitlán. Fuente: Elaboración propia con información de Semarnat (2014). 
La interpretación se realizó utilizando las clases de probabilidad mencionadas por Sánchez et al., (2004), que indican que un porcentaje de $0 \%$ a 33\% se considera como probabilidad baja de cambiar de una categoría a otra en un espacio de tiempo dado; mientras que un porcentaje de $34 \%$ a $66 \%$ es una probabilidad media y uno de $67 \%$ a 100\% representa una probabilidad alta (Castelán, Ruíz, Linares, Pérez y Tamaríz, 2007).

\section{Validación y precisión}

La validación del mapa de CT (2015) de la zona de estudio se llevó a cabo en diferentes pasos como a continuación se describe: a) se generó una malla sistemática de 564 puntos de control distribuidos de manera equidistante $(1000 \mathrm{~m}$ de separación) con la herramienta Create fishnet del programa ArcGis 10.2.2; b) a cada punto de la malla se le asignó una categoría de CT con base en el mapa de CT 2015; c) la malla de puntos fue trasladada al programa Google Earth Pro(C) en donde se le superpuso a una imagen satelital de la zona de estudio del mismo año; d) cada punto fue validado mediante fotointerpretación para determinar el acierto o desacierto de los puntos de control y establecer su precisión (Emanuelli, Duarte, Milla, Orellana y López, 2017).

\section{RESULTADOS Y DISCUSIÓN}

La distribución espacial de las categorías de CT para los años 2000 y 2015 sobre el territorio de Ixtacamaxtitlán se muestra en la figura 3. En el centro del municipio se observan áreas de pastizal que se han perdido al año 2015. Las superficies arboladas con mayor área se ubican en pendientes mayores a $50 \%$ de inclinación, a altitud superior a los $2700 \mathrm{~m}$ s.n.m. y de difícil acceso, distribuidas hacia el centro-sur del municipio, con fragmentos al norte y noreste. Las tierras de cultivo se encuentran en zonas con pendientes menores a $25 \%$ y altura menor a 2500 m s.n.m., así como en la planicie ubicada al centro del municipio; aunque, existen porciones distribuidas en toda el área de estudio.

La distribución en términos porcentuales y hectáreas de cada categoría de CT presentes en el municipio en los años 2000 y 2015 se especifica en la tabla 2. De las 56767 ha que componen la superficie total del área de estudio, la CT predominante en el año 2000 fue de superficies arboladas (36.8\%). Pese a que la categoría anteriormente mencionada presentó un aumento en distribución porcentual $(0.9 \%)$, para el año 2015 se convirtió en la segunda categoría predominante, por debajo de la de tierras de cultivo (38.38\%).
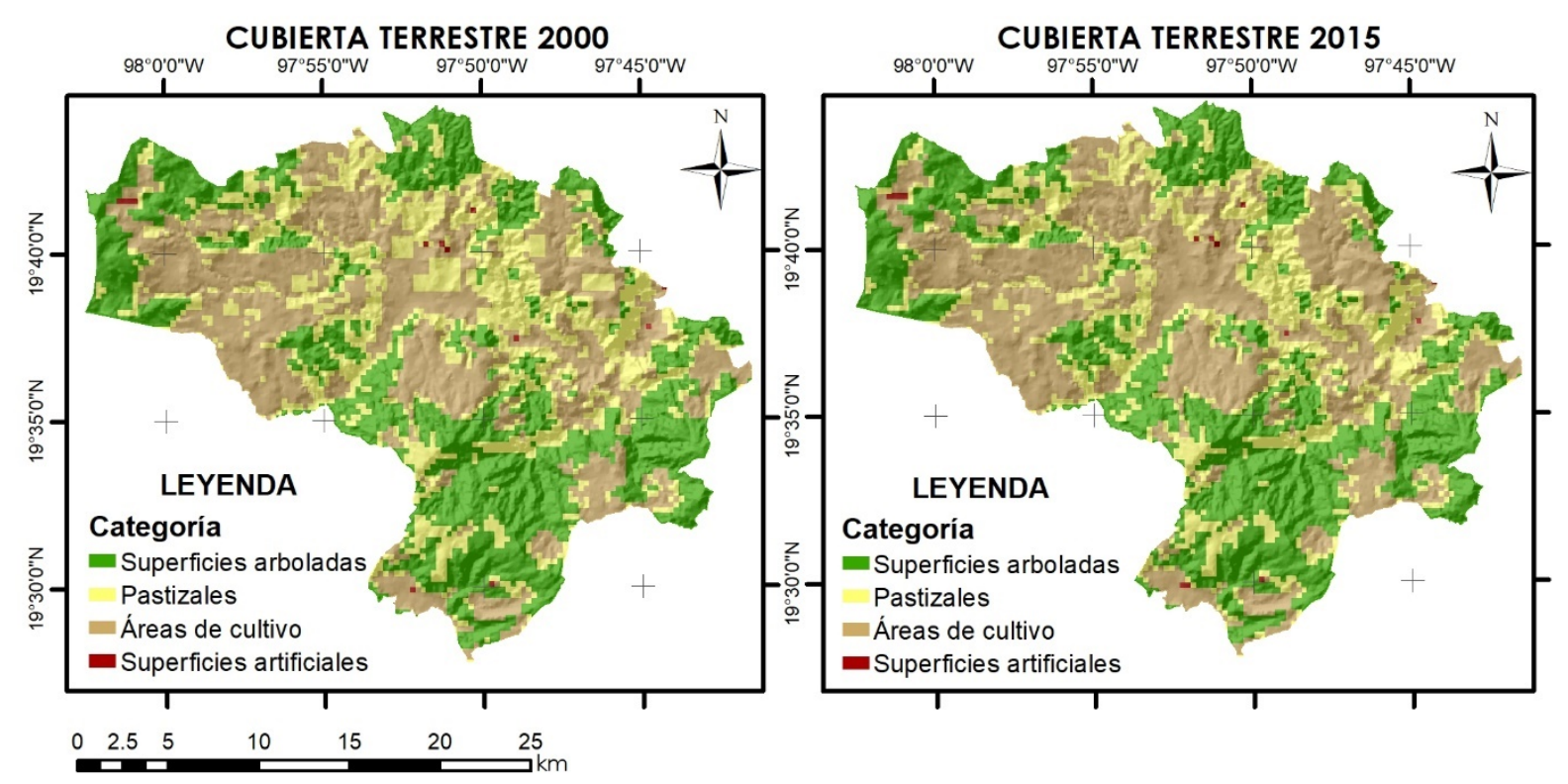

FIGURA 3: Distribución espacial de las categorías de cubierta terrestre sobre la zona de estudio para los años 2000 y 2015. Fuente: Elaboración propia con información de ESA. 
TABLA 2. Área de cada categoría de cubierta terrestre en los años 2000 y 2015.

\begin{tabular}{ccccc}
\hline & \multicolumn{2}{c}{2000} & \multicolumn{2}{c}{2015} \\
\cline { 2 - 5 } & ha & $\%$ & ha & 37.70 \\
\hline Superficies arboladas & 20890 & 36.8 & 20833 & 24.71 \\
Pastizales & 15387 & 27.11 & 14029 & 38.38 \\
Zonas de cultivo & 20379 & 35.90 & 21785 & 0.21 \\
Superficies artificiales & 111 & 0.20 & 120 & \\
\hline
\end{tabular}

Fuente: Elaboración propia.

En la tabla 3 se observan los procesos de cambio anuales sobre la CT, en donde se incluye el cambio neto en hectáreas y porcentaje para cada categoría y las tasas de cambio expresadas en porcentaje. La categoría de superficies arboladas presentó un cambio neto negativo de 57.2 ha $(0.27 \%)$; sin embargo, se observan fluctuaciones entre pérdidas y ganancias de superficie a lo largo del periodo de estudio, lo que puede indicar un proceso de deforestación acompañado de un nuevo crecimiento (Pontius et al., 2004). Otro cambio negativo se observó en los pastizales con una reducción de 1358 ha (8.83\%), dicha categoría ha perdido terreno en la superficie municipal cada año. Ambas categorías muestran una tasa de cambio anual menor a cero (-0.02\% y $-0.61 \%$, respectivamente), es decir, se presentaron reducciones en su superficie y los pastizales mostraron mayores decrementos.

Las categorías de tierras de cultivo y superficies artificiales presentan cambios netos positivos de 1407 ha y 9 ha, respectivamente, con tasas de cambio anuales mayores a cero $(0.45 \%$ y $0.52 \%)$, lo que reafirma los incrementos de estas en la superficie e indica que la categoría superficies artificiales presenta proporcionalmente mayor crecimiento en comparación con las demás categorías.

La baja pérdida de superficies arboladas puede deberse a los diferentes programas aplicados por parte del gobierno estatal para la reforestación (Gebhardt et al., 2014). Por ejemplo, en el año 2000, con el apoyo del gobierno, el municipio de Ixtacamaxtitlán, junto con otros ocho municipios, sumaron aproximadamente 3770 ha reforestadas, lo que representó $55 \%$ de la reforestación estatal; adicionalmente, en ese mismo año, a través del Programa Nacional de Reforestación (Pronare), fueron declaradas 153 ha como zonas de restauración ecológica (ZRE) en el municipio, debido a los incendios forestales ocurridos en 1998, con lo que se llevaron a cabo obras para la rehabilitación forestal (Comisión Nacional Forestal [Conafor], 2001). Ante este contexto, las comunidades de la zona de estudio registraron una alta participación en el aprovechamiento de los apoyos enfocados a la mejora de los bosques, lo que promovió la recuperación y restauración de la vegetación y el suelo (Conafor, 2002; Carabias, Arriaga y Cervantes-Gutiérrez, 2007). En la misma línea, en 2015, Ixtacamaxtitlán fue uno de los municipios del estado que tuvo mayor superficie reforestada con 299585 árboles plantados y distribuidos en 298 ha (Inegi, 2016).

La tendencia de los cambios anuales con los incrementos y reducciones en hectáreas de cada categoría se muestra en la figura 4. En las tierras de cultivo se presentó un incremento mayor a 100 ha en los años de 2001, 2008, 2010, 2011 y 2012; mientras que en los años 2005 y 2015 se presentó una reducción en esta categoría $(<10 \mathrm{ha})$. La reducción de pastizal mayor a 100 ha, en los años 2008, 2010 y 2011, ocurrió a la par de los incrementos de tierras de cultivo. Por otro lado, para el año 2006, la disminución de pastizal resultó en un incremento en las superficies arboladas. Otros incrementos en esta última categoría menores a 100 ha sucedieron en los años 2005 y 2008, mientras que reducciones importantes se observaron en 2001. 
TABLA 3. Datos anuales de cubierta terrestre y cambios netos de la superficie entre 2000-2015.

\begin{tabular}{|c|c|c|c|c|}
\hline \multirow{2}{*}{ Año } & \multicolumn{4}{|c|}{ Cubierta terrestre (ha) } \\
\hline & Superficies arboladas & Pastizales & Tierras de cultivo & Superficies artificiales \\
\hline 2000 & 20890 & 15387 & 20379 & 111 \\
\hline 2001 & 20785 & 15334 & 20537 & 111 \\
\hline 2002 & 20785 & 15334 & 20537 & 111 \\
\hline 2003 & 20740 & 15361 & 20555 & 111 \\
\hline 2004 & 20740 & 15361 & 20555 & 111 \\
\hline 2005 & 20767 & 15352 & 20537 & 111 \\
\hline 2006 & 20893 & 15218 & 20546 & 111 \\
\hline 2007 & 20904 & 15209 & 20543 & 111 \\
\hline 2008 & 20958 & 14719 & 20979 & 111 \\
\hline 2009 & 20958 & 14656 & 21042 & 111 \\
\hline 2010 & 20958 & 14244 & 21454 & 111 \\
\hline 2011 & 20958 & 14056 & 21642 & 111 \\
\hline 2012 & 20860 & 14029 & 21768 & 111 \\
\hline 2013 & 20833 & 14029 & 21794 & 111 \\
\hline 2014 & 20833 & 14029 & 21794 & 111 \\
\hline 2015 & 20833 & 14029 & 21785 & 120 \\
\hline Cambio neto (ha) & -57.2 & -1358 & +1407 & +9 \\
\hline Cambio neto (\%) & -0.27 & -8.83 & +6.90 & +8.11 \\
\hline Tasa de cambio (\%) & -0.02 & -0.61 & +0.45 & +0.52 \\
\hline
\end{tabular}

Fuente: Elaboración propia

Con lo descrito anteriormente se puede explicar que el detrimento de pastizales se relaciona con la expansión de superficies agrícolas. Dicha transición está dada por múltiples factores y depende de un contexto agrícola determinado (Martínez, 2017); en el caso de Ixtacamaxtitlán, la principal actividad económica ha sido la agricultura para autoconsumo, cuyos cultivos principales son: maíz, cebada, papa, frijol y trigo (Inegi, 2002), sin embargo en años recientes se ha observado una diversificación de cultivos a los que se suman avena, alberjones, calabaza, ajo, sorgo, haba, alfalfa, café cereza y naranja; aunque la mayoría de la producción agrícola es para autoconsumo, también se comercializa al interior de la región y en municipios cercanos, mostrando una actividad productiva variable (López, 2005; Bautista, Díaz, Estrada, 2007; Inegi, 2007; Tapia, 2018). 


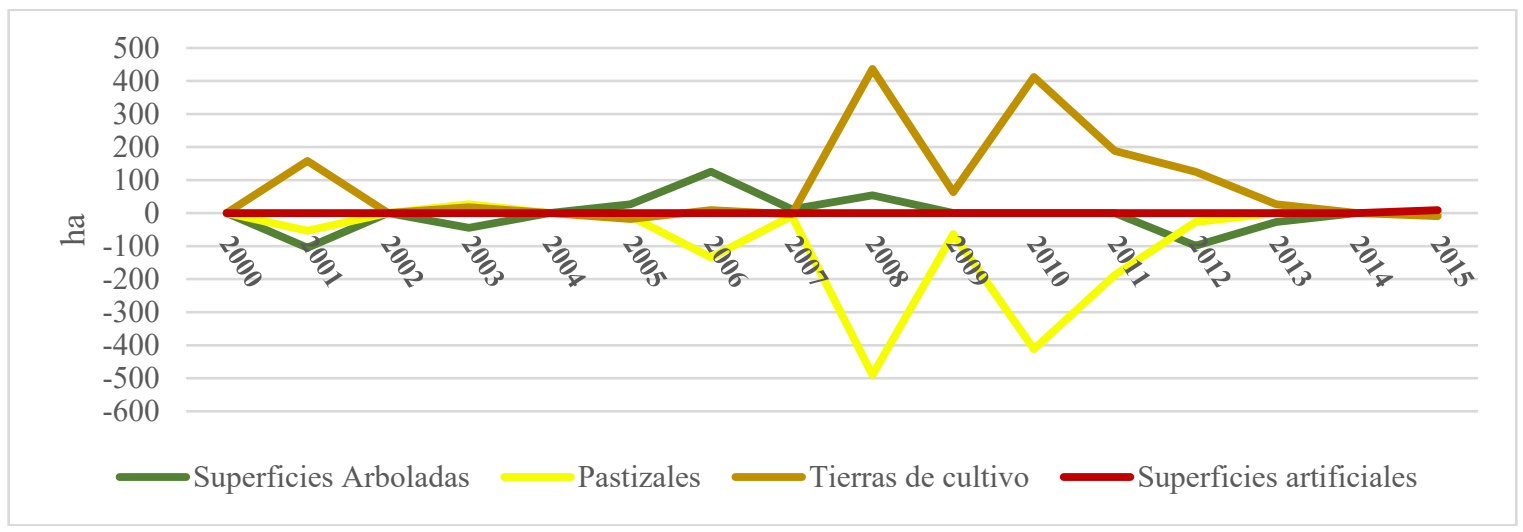

FIGURA 4: Cambios anuales de la cubierta terrestre en Ixtacamaxtitlán en el periodo 2000-2015.

En el eje Y se muestra la pérdida en hectáreas y en el eje $X$ los años de estudio.

Fuente: Elaboración propia

Aunado a esta situación, Ixtacamaxtitlán es uno de los municipios que pertenece a las Zonas de Atención Prioritaria (ZAP) por su alto grado de marginación (Consejo Nacional de Población [Conapo], 2012), por lo que forma parte de diversos apoyos, entre los que destacan el Programa de Atención a Jornaleros Agrícolas y el Programa $3 \times 1$ para Migrantes, este último cuenta con apoyo para proyectos productivos comunitarios y familiares para el desarrollo de las comunidades de origen de migrantes (Inegi, 2015); la migración es un fenómeno común en el municipio y se realiza principalmente a la Ciudad de México y a los Estados Unidos de Norteamérica (Bautista et al., 2007), en este sentido, cabe resaltar que la población tuvo una disminución de $13.4 \%$ al pasar de 28358 habitantes a 24512 en el periodo de estudio (Inegi, 2015); a pesar de esta disminución, hubo un aumento de población económicamente activa dedicada al sector primario, de $69.4 \%$ en el 2000 a $72.8 \%$ en el 2015 . Todos estos factores en su conjunto explican la expansión de tierras de cultivo en el territorio.

La matriz expresa los datos de tabulación cruzada entre las categorías de CT entre 2000 y 2015 y muestra la superficie total de tierra en hectáreas asociada con cada cambio, en donde el total de filas menos el valor diagonal que significa "sin cambios", representa las reducciones totales por categoría, mientras que el total de las columnas menos el valor diagonal representa los incrementos totales por categoría. Los datos de la matriz de cambios de CT (Tabla 4) muestran que 96.5\% (54 805 ha) de la superficie permaneció estable, por lo que, solo en 3.5\% (1962 ha) se registraron transiciones entre categorías. Los cambios negativos (reducciones) suman un total de 1715 ha, mientras que los cambios positivos (incrementos) fueron de 247 ha, estos cambios son atribuibles principalmente al paso de superficies de áreas naturales a actividades de producción.

El mayor incremento se produjo en las tierras de cultivo con 1569 ha, seguida de las superficies arboladas con 234 ha. Las categorías con menor incremento fueron los pastizales y las superficies artificiales con 150 ha y 9 ha, respectivamente. Las reducciones totales por categorías de mayor a menor son 1508 ha de pastizales, 291 ha de superficies arboladas y 163 ha de tierras de cultivo.

Los cambios positivos y negativos por categoría se muestran, como ganancias y pérdidas, en la tabla 4. Las 234 ha de incremento en las superficies arboladas son consideradas como ganancias, ya que son áreas forestadas que representan mejoras en los bienes y servicios ecosistémicos (Velázquez et al., 2002). 
TABLA 4. Matriz de cambios de la superficie 2000-2015.

\begin{tabular}{|c|c|c|c|c|c|c|c|}
\hline \multirow{8}{*}{$\begin{array}{l}\text { CT } 2000 \\
\text { (ha) }\end{array}$} & \multicolumn{7}{|c|}{ CT 2015 (ha) } \\
\hline & & $\begin{array}{c}\text { Superficies } \\
\text { Arboladas }\end{array}$ & Pastizales & $\begin{array}{c}\text { Tierras de } \\
\text { cultivo }\end{array}$ & $\begin{array}{l}\text { Superficies } \\
\text { artificiales }\end{array}$ & Reducción & Pérdida \\
\hline & $\begin{array}{l}\text { Superficies } \\
\text { arboladas }\end{array}$ & 20599 & 137 & 154 & 0 & 291 & 291 \\
\hline & Pastizales & 93 & 13879 & 1415 & 0 & 1508 & 1415 \\
\hline & $\begin{array}{l}\text { Tierras de } \\
\text { cultivo }\end{array}$ & 141 & 13 & 20216 & 9 & 163 & 9 \\
\hline & $\begin{array}{c}\text { Superficies } \\
\text { artificiales }\end{array}$ & 0 & 0 & 0 & 111 & 0 & 0 \\
\hline & Incremento & 234 & 150 & 1569 & 9 & $\begin{array}{c}\text { Sin } \\
\text { cambios }\end{array}$ & \\
\hline & Ganancia & 234 & 13 & 0 & 0 & & \\
\hline
\end{tabular}

Fuente: Elaboración propia.

Los pastizales se incrementaron en total 150 ha; sin embargo, únicamente 13 ha son consideradas ganancias, ya que pasaron de tierras de cultivo a pastizal; en este caso el proceso de cambio se considera como repliegue agrícola y podría estar dado por el abandono de tierras de cultivo debido a la disminución de los rendimientos agrícolas a causa de la erosión, lo que en muchos casos promueve que estas zonas se regeneren a su condición previa de pastizales o bosques (López, 2005; Bautista et al., 2007). El área restante (137 ha) es considerada como pérdida, ya que resultó en la reducción de 137 ha de superficies arboladas, por lo que se presentaron procesos de pérdida de cubierta forestal, posiblemente para la explotación maderable y el establecimiento de pastizal inducido para agostadero (López, 2005).

Los cambios en las tierras de cultivo únicamente se considerarán como ganancias si la transición proviniera de una superficie artificial; es decir, un proceso de expansión agrícola; sin embargo, esta transición no se presentó en la zona de estudio, más bien hubo pérdidas por deforestación, pérdida de vegetación diferente a la forestal y expansión agrícola, ya que los cambios se dieron de superficies arboladas y pastizales a tierras de cultivo (154 ha y 1415 ha , respectivamente), por lo que se consideran como procesos degenerativos (UNCCD, 2018). Otro proceso degenerativo presente, fue el crecimiento urbano en la zona de 9 ha, ya que significa la pérdida irremediable del suelo y la vegetación a causa del sellado.

En la figura 5 se muestra la distribución espacial de los cambios (positivos y negativos) y los flujos (ganancias o pérdidas por categoría) que se presentaron en la zona de estudio (2000-2015). Se observa que la mayoría de los cambios negativos, así como las pérdidas de pastizales, se presentaron en el centro-norte del municipio, lo que probablemente se explique por la mayor accesibilidad que se tiene en esa zona.

Todas las categorías superan $90 \%$ de probabilidad de permanencia, por lo que esta se considera como alta (Fig. 6); es decir, pese a los diversos procesos de cambio, existe una baja probabilidad de transformaciones en la CT en la zona de estudio, lo que podría estar causado como se mencionó anteriormente, por la disminución de la población en el periodo, así como la falta de vialidades en la zona que dificulta la comunicación y comercialización. La probabilidad de permanencia de las superficies urbanas es de $100 \%$ y, aunque existe cierta probabilidad de transformación de tierra de cultivo a superficie urbana, esta es mínima $(0.04 \%)$ por lo que no fue considerada dentro del diagrama. 


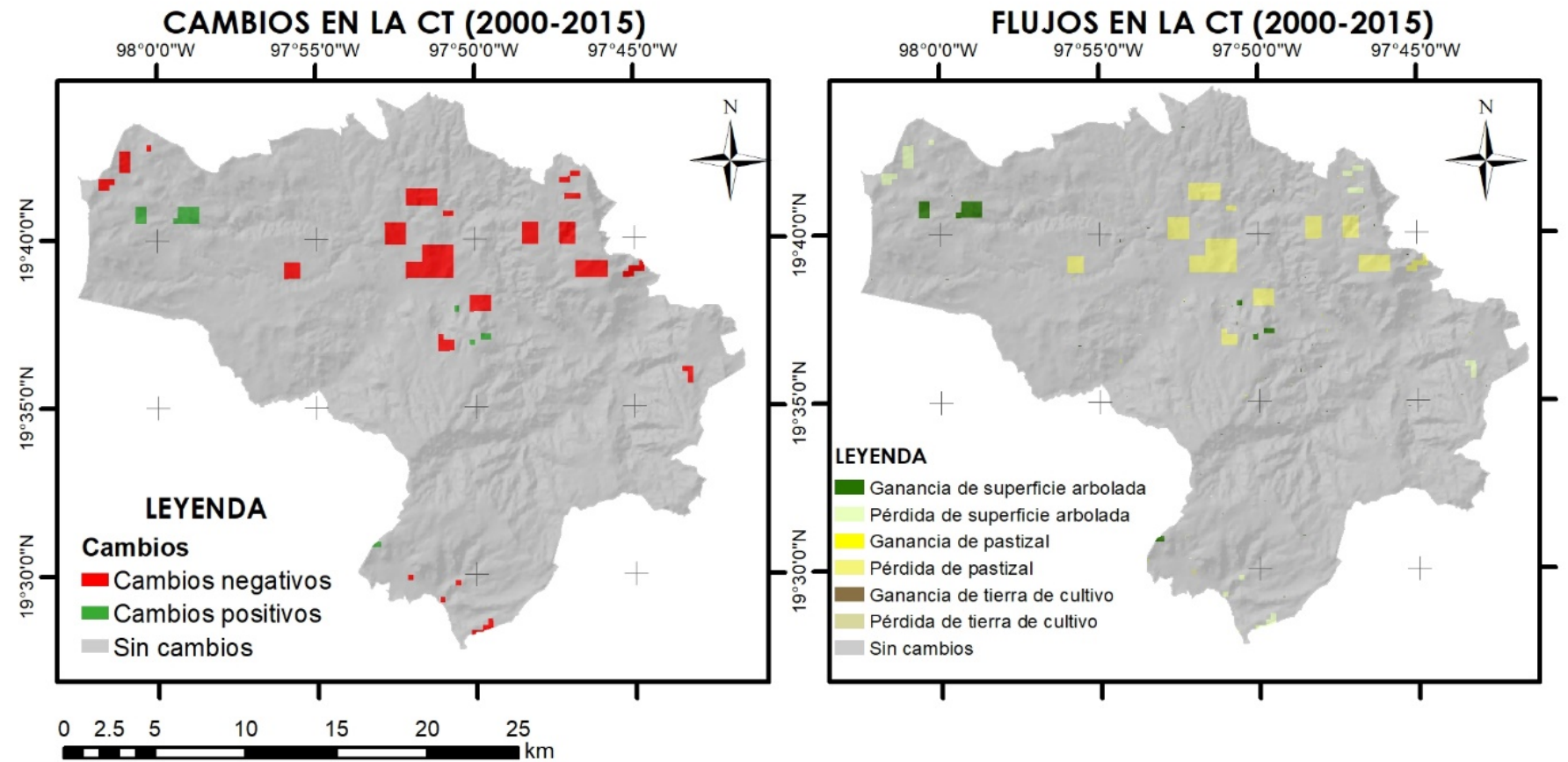

FIGURA 5: Mapa de cambios y flujos en la cubierta terrestre sobre el territorio municipal 2000-2015.

Fuente: Elaboración propia

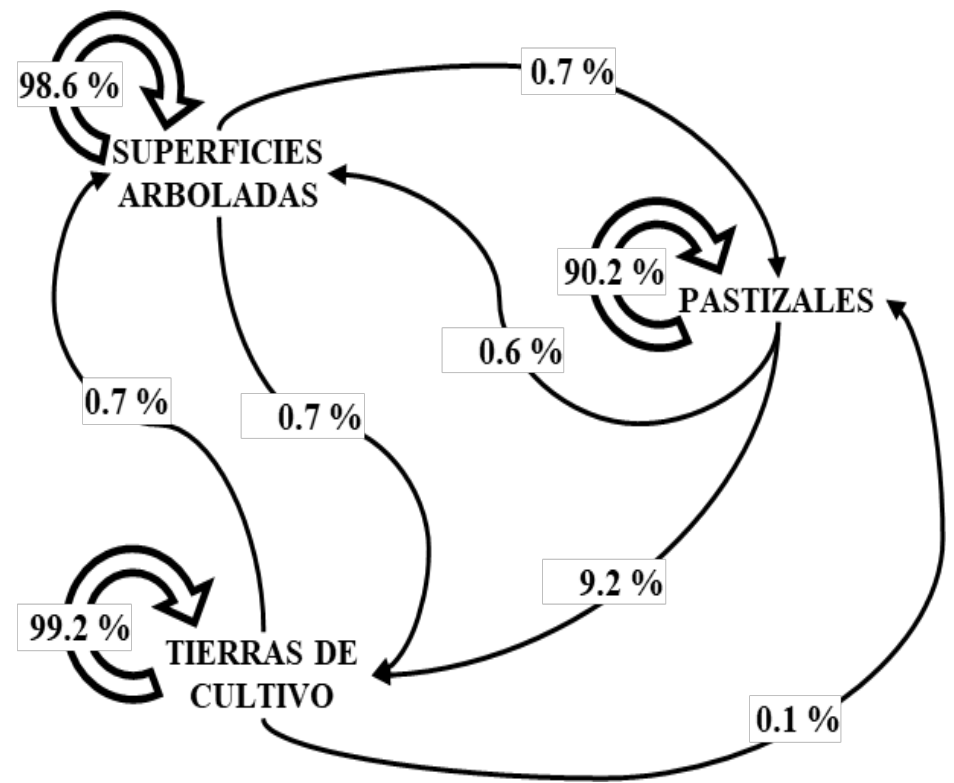

FigURA 6: Diagrama de transformaciones sobre la cubierta terrestre de Ixtacamaxtitlán, Puebla en el periodo del 2000-2015 y su probabilidad de permanencia $(\%)$.

Fuente: Elaboración propia 
El análisis comparativo entre los puntos validados y el mapa de CT de 2015 mostró una precisión de 85.64\% y un error de $14.36 \%$, de un total de 564 puntos evaluados (Tabla 5). $\mathrm{Si}$ bien la resolución espacial puede perder algunos de los detalles importantes para escalas estatales y municipales (subnacionales), el conjunto de datos de CCL-LC de ESA presentó un buen nivel de precisión a escala municipal, ya que rebasa el porcentaje mínimo permitido para la validación cartográfica, la cual corresponde a $80 \%$ (Camacho-Sanabria et al., 2017, Emanuelli et al., 2017) y que, en el caso de las series de uso de suelo y vegetación de Inegi ubican su precisión a escala nacional entre 74\% y 89\% (Mas, Velázquez y Couturier, 2009).

TABLA 5. Precisión y error del mapa de cubierta terrestre 2015.

\begin{tabular}{cc}
\hline Estadístico & Conteo \\
\hline Aciertos & 483 \\
Desaciertos & 81 \\
Total de puntos & 564 \\
Precisión (\%) & 85.64 \\
Error (\%) & 14.36 \\
\hline
\end{tabular}

Fuente: Elaboración propia

\section{CONCLUSIONES}

De acuerdo con los resultados obtenidos, en el periodo de 15 años (2000-2015) las categorías de CT en el municipio presentaron diferentes procesos de transformación. En el caso de las superficies arboladas y los pastizales presentaron decrementos de $0.27 \%$ y $8.83 \%$, respectivamente. Mientras que las tierras de cultivo y superficies urbanas presentaron incrementos de $6.9 \%$ y $8.11 \%$. Espacialmente, la mayoría de los cambios se concentraron principalmente en las zonas de fácil acceso presentes en el centro-norte del municipio.

La mayor parte del territorio municipal $(96.5 \%)$ se ha mantenido estable, lo que determinó que las categorías de CT tengan alta probabilidad de permanencia; sin embargo, $3.5 \%$ de la superficie presentó transiciones o intercambios entre categorías. Los cambios negativos sumaron 87.4\%, mientras que los cambios positivos fueron de 12.6\%. En este sentido, resaltan como los cambios más importantes, las pérdidas de pastizales convertidas a tierras de cultivo (1415 ha) y los intercambios entre superficies arboladas y tierras de cultivo, que se han mantenido relativamente estables.

Las transformaciones de la superficie se deben a diversos procesos, entre los cuales destacan los programas a escala estatal que han promovido que las superficies arboladas se mantengan desde el año 2000. Así mismo, la diversificación del cultivo y la actividad productiva variable en la zona de estudio pueden explicar la disminución de pastizales debido al aumento de tierras de cultivo. De igual manera, las dinámicas socioeconómicas de la población en la zona de estudio como la disminución de la población, la migración y el aumento de personas dedicadas al sector primario pueden intervenir en los procesos de cambio.

Se concluye que el conjunto de datos de ESA-CCI-LC tiene una alta precisión $(>85 \%)$ a escala subnacional y permite la visualización de las transformaciones más importantes ocurridas sobre la superficie del territorio de Ixtacamaxtitlán.

\section{REFERENCIAS}

Bautista, S., Díaz, C., \& Estrada, M. (2007). Producción avícola familiar en una comunidad del municipio de Ixtacamaxtitlán, Puebla. Revista Mexicana de Ciencias Pecuarias, 45(1), 41-60.

Camacho-Sanabria, R., Camacho-Sanabria, J., Balderas-Plata, M., \& Sánchez-López, M. (2017). Cambios de cobertura y uso de suelo: estudio de caso en Progreso Hidalgo, Estado de México. Madera y Bosques, 23(3), 39-60. doi: 10.21829/myb.2017.2331516

Carabias, J., Arriaga, V., \& Cervantes-Gutiérrez, V. (2007). Las políticas públicas de la restauración ambiental en México: limitantes, avances, rezagos y retos. Botanical Sciences, 80(S), 85-100. doi: $10.17129 /$ botsci.1759

Castelán, R., Ruiz, J., Linares, G., Pérez, R., \& Tamariz, V. (2007). Dinámica de cambio espacio-temporal de uso del suelo de la subcuenca del río San Marcos, Puebla, México. Investigaciones Geográficas, (64), 75-89.

Comisión Nacional Forestal [Conafor]. (2001). Evaluación del Programa Nacional de Reforestación (Pronare 2000-2001) Puebla. Unidad de 
investigación, capacitación y evaluación para el desarrollo rural de la Universidad Autónoma de Chapingo. México: Conafor.

Comisión Nacional Forestal [Conafor]. (2002). Evaluación del Programa Nacional de Reforestación (Pronare 2002) Puebla. Unidad de investigación, capacitación y evaluación para el desarrollo rural de la Universidad Autónoma Chapingo. México: Conafor. Recuperado de https://n9.cl/p4h7

Consejo Nacional de Población [Conapo]. (2012). Índice de marginación por localidad. Índices sociodemográficos. México: Conapo. Recuperado de http://www.conapo.gob.mx/es/CONAPO/Indice_de_Margina cion_por_Localidad_2010

Defourny, P., Bontemps, S., Lamarche, C., Brockmann, C., Boettcher, M., Wevers, J., \& Kirches, G. (2017). Land cover CCI: product user guide version 2.0. Recuperado de https://maps.elie.ucl.ac.be/CCI/viewer/download/ESACCILC-Ph2-PUGv2_2.0.pdf

Dewan, A., Yamaguchi, Y., \& Rahman, M. (2012). Dynamics of land use/cover changes and the analysis of landscape fragmentation in Dhaka Metropolitan, Bangladesh. GeoJournal 77(3), 315-330. doi: 10.1007/s1070 8-010-9399-x

Emanuelli, P., Duarte, E., Milla, F., Orellana, O., \& López, S. (2017). Sitio piloto Cantón de Puriscal. Validación del mapa de cobertura forestal y uso de la tierra del año 2012. doi: 10.13140/RG.2.2.32894.59204

Environmental Systems Research Institute [Esri]. (2014). ArcGIS Desktop 10.2.2. Redlands, E.U.

Feddema, J., Oleson, K., Bonan, G, Mearns, L., Buja, L., Meehl, G., \& Washington, W. (2005). The importance of land-cover change in simulating future climates. Science, 310(5754), 1674-1678. doi: 10.1126/science. 1118160

Food and Agriculture Organization [FAO]. (1996). Forest resources assessment 1990. Survey of tropical forest cover and study of change processes. Roma: FAO. Recuperado de http://www.fao.org/3/w0015e/W0015E00.htm

Food and Agriculture Organization [FAO] (2016). Land Cover Classification System (LCCS). Classification concepts. Software version 3. Roma: FAO. Recuperado de http://www.fao.org/3/ai5232e.pdf

Fritz, S., See, L., McCallum, I., Schill, C., Obersteiner, M., Van der Velde, M., Boettcher, H., Havlik., \& Achard, F. (2011). Highlighting continued uncertainty in global land cover maps for the user community. Environmental Research Letters, 6(4), 044005. doi: 10.1088/1748-9326/6/4/044005

Gebhardt, S., Wehrmann, T., Muñoz-Ruiz, M., Maeda, P., Bishop, J., Schramm, M., Kopeinig, R., Cartus, O., Kellndorfer, J., Ress, R., Santos, L., \& Schmidt, M. (2014). MAD-MEX: automatic wall-to- wall land cover monitoring for the Mexican REDD-MRV program using all Landsat data. Remote Sensing, 6(5), 3923-3943. doi: $10.3390 /$ rs6053923

Gichenje, H., \& Godinho, S. (2018). Establishing a Land Degradation Neutrality national baseline through trend analysis of GIMMS NDVI Time-series. Land Degradation \& Development, 29(9), 29852997. doi: 10.1002/ldr.3067

Grekousis, G., Mountrakis, G., \& Kavouras, M. (2015). An overview of 21 global and 43 regional land-cover mapping products. International Journal of Remote Sensing, 36(21), 5309-5335. doi: 10.1080/01431161.2015.1093195

Guidigan, M., Sanou, C., Ragatoa, D., Fafa, C., \& Mishra, V. (2019). Assessing land use/land cover dynamic and its impact in Benin Republic using land change model and CCI-LC products. Earth Systems and Environment, 3(1), 127-137. doi: 10.1007/s41748-0180083-5

Instituto Nacional de Estadística y Geografía [Inegi]. (2002). Anuario estadístico del estado de Puebla, 2002. Aguascalientes, México: Inegi. Recuperado de https://n9.cl/21qth

Instituto Nacional de Estadística y Geografía [Inegi]. (2007). III Censo agricola, ganadero y forestal 2007. México: Inegi. Recuperado de https://www.inegi.org.mx/programas/cagf/2007/

Instituto Nacional de Estadística y Geografía [Inegi]. (2009). Prontuario de información geográfica municipal de los Estados Unidos Mexicanos. Ixtacamaxtitlán, Puebla. México: Inegi. Recuperado de https://n9.cl/hbqx1

Instituto Nacional de Estadística y Geografía [Inegi]. (2014). Conjunto de datos vectoriales edafológico, escala 1:250000, serie II. (Continuo nacional). Aguascalientes. México: Inegi. Recuperado de http://www.conabio.gob.mx/informacion/gis/

Instituto Nacional de Estadística y Geografía [Inegi]. (2015). Información nacional, por entidad federativa y municipios. Ixtacamaxtitlán, Puebla. México: Inegi. Recuperado de http://www.beta.inegi.org.mx/app/areasgeograficas/default.asp $\mathrm{x}$

Instituto Nacional de Estadística y Geografía [Inegi]. (2016). Anuario estadístico y geográfico de Puebla 2016. México: Inegi. Recuperado de https://n9.cl/ch4bq

Intergovernmental Panel on Climate Change [IPCC]. (2006). IPCC guidelines for national greenhouse gas inventories. Recuperado de https://www.ipcc-nggip.iges.or.jp/public/2006gl/

Jiang, L., \& Yu, L. (2019). Analyzing land use intensity changes within and outside protected areas using ESA CCI-LC datasets. Global Ecology and Conservation, 20(2019), e00789. doi: 10.1016/j.gecco.2019.e00789 
Li, W., Ciais, P., MacBean, N., Peng, S., Defourny, P., \& Bontemps, S. (2016). Major forest changes and land cover transitions based on plant functional types derived from the ESA CCI Land Cover product. International Journal of Applied Earth Observation and Geoinformation, 47, 30-39. doi: 10.1016/j.jag.2015.12.006

Li, W., MacBean, N., Ciais, P., Defourny, P., Lamarche, C., Bontemps, S., Houghton, R., \& Peng, S. (2018). Gross and net land cover changes in the main plant functional types derived from the annual ESA CCI Land Cover maps (1992-2015). Earth System Science Data, 10(1): 219-234. doi: 10.5194/essd-10-219-2018

Liu, X., Yu, L., Li, W., Peng, D., Zhong, L., Li, L., Xin, Q., Lu, H., Yu, C., \& Gong, P. (2018). Comparison of country-level cropland areas between ESA-CCI land cover maps and FAOSTAT data. International Journal of Remote Sensing, 39(20), 6631-6645. doi: 10.1080/01431161.2018.1465613

López, C. (2005). Ganadería campesina en agostaderos de uso común: estudio de caso en Ixtacamaxtitlán, Puebla. Tesis de maestría. Programa en Estrategias para el Desarrollo Agrícola Regional. Colegio de Postgraduados Campus Puebla, Puebla, México. Recuperado de https://n9.cl/t2mz

Martínez, C. (2017). El abandono de campos de cultivo en la Región de Murcia: causas y consecuencias medioambientales y socioeconómicas. Tesis de doctorado. Universidad de Murcia, Murcia, España. Recuperado de https://www.tdx.cat/handle/10803/405714

Mas, J. F., Velázquez, A., \& Couturier, S. (2009). La evaluación de los cambios de cobertura/uso del suelo en la República Mexicana. Investigación Ambiental Ciencia y Politica Pública, 1(1), 23-39.

Mousivand, A., \& Arsanjani, J. (2019). Insights on the historical and emerging global land cover changes: The case of ESA-CCI-LC datasets. Applied Geography, 106, 82-92. doi: 10.1016/j.apgeog.2019.03.010

Orr, B., Cowie, A., Castillo, V., Chasek, P., Crossman, N., Erlewein, A., Louwagie, G., Maron, M., Metternicht, G., Minelli, S., Tengberg, A., Walter, S., \& Welton, S. (2017). Scientific conceptual framework, for Land Degradation Neutrality. A report of the science-policy interface. United Nations Convention to Combat Desertification (UNCCD), Bonn, Germany.

Peralta-Carreta, C., Gallardo-Cruz, J., Solórzano, J., \& HernándezGómez, M. (2019). Clasificación del uso de suelo y vegetación en áreas de pérdida de cobertura arbórea (2000-2016) en la cuenca del río Usumacinta. Madera y Bosques, 25(3), e2531779. doi: $10.21829 /$ myb.2019.2531779

Programa Mundial de Apoyo (PMA). (2016). Programa para el establecimiento de metas en la Neutralidad de la Degradación de las Tierras (NDT). Nota metodológica para el establecimiento de metas nacionales voluntarias para la Neutralidad en la Degradación de las Tierras haciendo uso del marco de indicadores de la UNCCD. Bonn, Germany. Recuperado de https://n9.cl/qkfqv

Pontius, R., Shusas, E., \& McEachern, M. (2004). Detecting important categorical land changes while accounting for persistence. Agriculture Ecosystems y Environment, 101(2-3), 251-268. doi: 10.1016/j.agee.2003.09.008

Sánchez, E. (2015). Manejo del suelo en la zona de restauración ecológica Texocuixpan, municipio de Ixtacamaxtitlán. Tesis de licenciatura. Universidad Autónoma Agraria Antonio Narro. Saltillo, Coahuila, México. Recuperado de http://repositorio.uaaan.mx:8080/ xmlui/handle/123456789/5738

Sánchez, J., Bocco, G., Fuentes, J. \& Velázquez, A. (2004). Análisis de cobertura y uso del terreno en el contexto de su dinámica espaciotemporal. En Velázquez, A., Torres, A., \& Bocco, G. (Comps), Las enseñanzas de San Juan: investigación participativa para el manejo integral de recursos naturales. (pp. 235-256). Secretaría de Medio Ambiente y Recursos Naturales. Instituto Nacional de Ecología. México. Recuperado de https://n9.cl/8u2ek

Secretaría de Medio Ambiente y Recursos Naturales [Semarnat]. (2013). Inventario estatal forestal y de suelos - Puebla 2013. México: Semarnat.

Secretaría de Medio Ambiente y Recursos Naturales [Semarnat]. (2014) Diagnóstico del Programa de Manejo de Tierras para la Sustentabilidad Productiva (PNACDD). Dirección General del Sector Primario y Recursos Naturales Renovables. México: Semarnat. Recuperado de

https://www.coneval.org.mx/Informes/Evaluacion/Diagnostic o/Diagnostico_2014/Diagnostico_2014_SEMARNAT_F002.p df

Tapia, L. (2018). Infraestructura carretera y economía regional. El caso de los productores de ajo de Oaxaca y Puebla. Región y Sociedad, $30(73)$.

United Nations Convention to Combat Desertification [UNCCD]. (2018). Default data: methods and interpretation. A guidance document for 2018 UNCCD reporting. United Nations Convention to Combat Desertification (UNCCD), Bonn, Germany. Recuperado de https://prais.unccd.int/sites/default/files/helper_documents/3 -DD_guidance_EN.pdf

United Nations Convention to Combat Desertification [UNCCD]. (2016). Achieving Land Degradation Neutrality at the country level. Building blocks for LDN target setting. Recuperado de https://www.unccd.int/sites/default/files/documents/1810201 6_LDN\%20country\%20level_ENG.pdf

Velázquez, A., Mas, J. F., Gallegos, J. D., Saucedo, R. M., Alcántara, P. C., Castro, R., Fernandez, T., Bocco, G., Ezcurra, E., \& Palacio, J. L. (2002). Patrones y tasas de cambio de uso del suelo en México. Gaceta Ecológica, (62), 21-37. 
Manuscrito recibido el 14de julio de 2020

Aceptado el 14 de enero de 2021

Publicado el 30 de diciembre de 2021

Este documento se debe citar como:

Arroyo-Ortega, I., Tamaríz-Flores, V., Torres-Ramírez, E., Handal-Silva, A., Chávez-Bravo, E., Cervantes-Gutiérrez, V., \& Castelán-Vega, R. (2021). Cambios en la cubierta terrestre a través de los mapas ESA-CCI-LC (2000-2015), Ixtacamaxtitlán, Puebla. Madera y Bosques, 27(3), e2732210. doi: 10.21829/myb.2021.2732210

Madera y Bosques por Instituto de Ecología, A.C. se distribuye bajo una Licencia Creative Commons Atribución-NoComercialCompartirlgual 4.0 Internacional. 